\title{
Optical-Model Potential and Broad Single-Particle Resonances
}

\author{
J. CUGNON * \\ Theoretical Nuclear Physics, University of Liège, \\ Sart Tilman, 4000 Liège 1 , Belgium
}

Received November 6, 1969

The dynamical energy dependence of the optical-model potential is studied in the case of a broad single-particle resonance. It is found that the optical-model potential is slowly energy-varying. The optical phase shift and the transmission coefficient are shown to display a resonance.

\section{Introduction}

The main purpose of this paper is to study the energy-dependence of the optical-model potential in the presence of a broad single-particle resonance. We work in the frame of the shell-model approach to nuclear reactions ${ }^{1}$. The optical-model potential obtained in this way is nonlocal and energy-dependent. This energy-dependence is called ${ }^{2}$ the dynamic energy dependence of the optical-model potential. If one replaces the non-local potential by an equivalent local one, (this procedure is required in order to connect theoretical and phenomenological opticalmodel potentials) the energy-dependence is modified. Here we are only interested in the dynamic energy dependence.

It is found that the dynamic energy dependence of the optical-model potential in the vicinity of a broad single-particle resonance is negligible, while the optical phase shift is strongly varying and exhibits a resonance. We explain this phenomena by deriving a simple relation which gives the connection between the imaginary part of the optical potential, the optical phase shift and the absorption in one channel. This relation emphasizes that the optical phase shift and the absorption depend upon the real as well as the imaginary parts of the complex potential. The imaginary part of the optical phase shift and the absorption display a resonance when the phase shift due to the real part of the optical-model potential displays a resonance or when the imaginary part has a BreitWigner form. The first case occurs in the presence of a broad singleparticle resonance, the second one in the case of a doorway state.

* Chercheur I.I.S.N.

1 Mahaux, C., Weidenmüller, H. A.: Shell-model approach to nuclear reactions. Amsterdam: North-Holland Publ. Co. 1969.

2 Lipperheide, R., Schmidt, A. K.: Nucl. Phys. A112, 65 (1968).

10 Z. Physik, Bd. 233 
In Section 2, we derive formulae for the generalized optical-model potential in channel $c(\operatorname{GOMP}(c))$, which is defined to be a one-body potential the scattering function of which is equal to the diagonal matrix element in channel $c$ of the collision matrix. In Section 3, we define the optical-model potential in channel $c(\mathrm{OMP}(c))$ and derive its expression. Those formulae are valid if no narrow single-particle resonance is present. We study the behaviour of $\mathrm{OMP}(c)$ through a broad s.-p. resonance in Section 4. We also establish a relation between the optical model potential, the "strength function" and the optical phase shift in a simple case. The introduction of the channel-channel coupling is examined. Section 5 contains the conclusions.

\section{The GOMP(c)}

Several methods have been proposed to construct the $\operatorname{GOMP}(c)^{1,3-5}$ from a microscopic point of view. We will generalize here the method of Mahaux and Weidenmüller ${ }^{1}$ to the case where a single-particle resonance exists. The Hamiltonian of the system has the form

$$
H=H_{0}+V \text {, }
$$

where $H_{0}$ is an independent particle Hamiltonian and $V$ a residual interaction. The Hamiltonian $H_{0}$ possesses bound states $\Phi_{i}$ and scattering states $\chi_{E}^{c}$ which describe $A-1$ nucleons in a bound state $\Omega_{c}$ and the $A$-th nucleon in a scattering state $u_{l j}\left(r, k_{c}\right)$. The latter function satisfies the equation

$$
\left(E-D_{l j}\right) u_{l j}\left(r, k_{c}\right)=0,
$$

where $D_{l j}$ is the radial part of the single-particle hamiltonian. We introduce the equations and definitions

$$
\begin{aligned}
& H_{0} \Phi_{j}=E_{j} \Phi_{j}, \quad H_{0} \chi_{E}^{c}=E \chi_{E}^{c}, \\
& \left\langle\Phi_{i} \mid \Phi_{j}\right\rangle=\delta_{i j}, \quad\left\langle\Phi_{i} \mid \chi_{E}^{c}\right\rangle=0, \quad\left\langle\chi_{E}^{c} \mid \chi_{E^{\prime}}^{c^{\prime}}\right\rangle=\delta_{c c^{\prime}} \delta\left(E-E^{\prime}\right), \\
& \left\langle\Phi_{i}|V| \Phi_{j}\right\rangle=V_{i j}, \quad\left\langle\Phi_{i}|V| \chi_{E}^{c}\right\rangle=V_{i}^{c}(E), \\
& \left\langle\chi_{E^{\prime}}^{c^{\prime}}|V| \chi_{E^{\prime \prime}}^{c^{\prime \prime}}\right\rangle=V\left(E^{\prime}, E^{\prime \prime}, c^{\prime}, c^{\prime \prime}\right) .
\end{aligned}
$$

The Lippmann-Schwinger equations reads ${ }^{1}$

$$
\Psi_{E}^{c(+)}=\chi_{E}^{c(+)}+\frac{1}{E^{+}-H_{0}} V \Psi_{E}^{c(+)} .
$$

3 Feshbach, H.: Ann. Phys. 5, 357 (1958); 19, 287 (1962).

4 Lipperheide, R.: Nucl. Phys. 89, 97 (1966).

5 Giai, N. van, Sawicki, J., Vinh Mau, N.: Phys. Rev. 141, 913 (1966). 
The function $\Psi_{E}^{c(+)}$ has an incoming wave in the channel $c$ only. Writing it in the form

$$
\Psi_{E}^{c(+)}=\sum_{i=1}^{M} b_{E}^{c}(i) \Phi_{i}+\sum_{c^{\prime} \varepsilon_{c^{\prime}}}^{\infty} a_{E}^{c}\left(E^{\prime}, c^{\prime}\right) \chi_{E^{\prime}}^{c^{\prime}} d E^{\prime},
$$

and taking account of Eq. (2.3), one obtains the system

$$
\begin{aligned}
& \left(E-E_{i}\right) b_{E}^{c}(i)-\sum_{j=1}^{M} V_{i j} b_{E}^{c}(j)-\sum_{c^{\prime} \varepsilon_{c^{\prime}}}^{\infty} d E^{\prime} a_{E}^{c}\left(E^{\prime}, c^{\prime}\right) V_{i}^{c^{\prime}}\left(E^{\prime}\right)=0, \\
& \left(E-E^{\prime}\right) a_{E}^{c}\left(E^{\prime}, c^{\prime}\right) \\
& -\sum_{m=1}^{M} V_{m}^{c^{\prime}}\left(E^{\prime}\right) b_{E}^{c}(m)-\sum_{c^{\prime \prime} \delta_{c^{\prime \prime}}}^{\infty} d E^{\prime \prime} a_{E}^{c}\left(E^{\prime \prime}, c^{\prime \prime}\right) V\left(E^{\prime \prime}, E^{\prime}, c^{\prime \prime}, c^{\prime}\right)=0 .
\end{aligned}
$$

The quantities $\varepsilon_{c^{\prime}}$ are the threshold energies. If $V\left(E^{\prime \prime}, E^{\prime}, c^{\prime \prime}, c^{\prime}\right)=0$, the kernel of the $L-S$ equation is of finite rank, and the system (2.6) can be solved by algebraic methods. The $S$ matrix is then given by

$$
S_{c c^{\prime}}=\exp \left\{i\left(\delta_{c}+\delta_{c^{\prime}}\right)\right\}\left\{1-i 2 \pi \sum_{j, m=1}^{M} V_{j}^{c}(E)\left[D^{-1}(E)\right]_{j m} V_{m}^{c^{\prime}}(E)\right\}
$$

where $\delta_{c}$ is the potential phase shift, while the matrix $D$ is given by

with

$$
[D(E)]_{j m}=\left\langle\Phi_{j}\left|E-H-V \sum_{c^{\prime}} G_{0}^{c^{\prime}}(E) V\right| \Phi_{m}\right\rangle
$$

$$
G_{0}^{c^{\prime}}(E)=\int_{\varepsilon_{c^{\prime}}}^{\infty} \frac{\left.\chi_{E^{\prime}}^{c^{\prime}}\right\rangle d E^{\prime}\left\langle\chi_{E^{\prime}}^{c^{\prime}}\right.}{E^{+}-E^{\prime}}
$$

In the case $V\left(E^{\prime \prime}, E^{\prime}, c^{\prime \prime}, c^{\prime}\right)=0$, the construction of $\operatorname{GOMP}(c)$ is very simple. Eliminating the coefficients $b_{E}^{c}(j)$ and $a_{E}^{c}\left(E^{\prime}, c^{\prime}\right)$ for $c^{\prime} \neq c$ in Eq. (2.6), one obtains

with

$$
\left(E-E^{\prime}\right) a_{E}^{c}\left(E^{\prime}, c\right)-\int_{\varepsilon_{c}}^{\infty} d E^{\prime \prime} \mathscr{V}_{E}^{c}\left(E^{\prime}, E^{\prime \prime}\right) a_{E}^{c}\left(E^{\prime \prime}, c\right)=0,
$$

$$
\begin{aligned}
\mathscr{V}_{E}^{c}\left(E^{\prime}, E^{\prime \prime}\right) & =\sum_{j, m=1}^{M} V_{j}^{c}\left(E^{\prime}\right)\left[{ }^{c} D^{-1}(E)\right]_{j m} V_{m}^{c}\left(E^{\prime \prime}\right), \\
{\left[{ }^{c} D(E)\right]_{j m} } & =\left\langle\Phi_{j}\left|E-H-V \sum_{c^{\prime} \neq c} G_{0}^{c^{\prime}}(E) V\right| \Phi_{m}\right\rangle .
\end{aligned}
$$

Multiplying Eq. (2.10) by the function $u_{l j}\left(r, k_{c}\right)$ and integrating over the energy, one gets, using Eq. (2.2):

$$
\left(E-D_{l j}\right) \rho_{E}^{c}(r)=\int_{0}^{\infty} d r^{\prime} \mathscr{F}_{E}^{c}\left(r, r^{\prime}\right) \rho_{E}^{c}\left(r^{\prime}\right),
$$


where

$$
\begin{aligned}
\rho_{E}^{c}(r) & =\int_{\varepsilon_{c}}^{\infty} d E^{\prime} a_{E}^{c}\left(E^{\prime}, c\right) u_{l j}\left(r, k_{c}^{\prime}\right), \\
\mathscr{V}_{E}^{c}\left(r, r^{\prime}\right) & =\sum_{j, m=1}^{M} V_{j}^{c}(r)\left[{ }^{c} D^{-1}(E)\right]_{j m} V_{m}^{c}\left(r^{\prime}\right), \\
V_{j}^{c}(r) & =\int_{\varepsilon_{c}}^{\infty} V_{j}^{c}(E) u_{l j}\left(r, k_{c}\right) d E .
\end{aligned}
$$

The quantity $\rho_{E}^{c}(r)$ is the projection on channel $c$ of the scattering (unbound) part of the function $\Psi_{E}^{c(+)}$.

If $V\left(E^{\prime \prime}, E^{\prime}, c^{\prime \prime}, c^{\prime}\right) \neq 0$, the kernel of the Lippmann-Schwinger equation is not of finite rank. One can write the kernel in the form

$$
K=K_{1}+K_{s}
$$

with

$$
K_{s}=\frac{1}{E^{+}-H_{0}} V_{s}
$$

Here, $V_{s}$ is the part of $V$ acting in the space of the functions $\chi_{E}^{c}$. The resolvent of $K$ may be calculated if the resolvent of $K_{s}$ is known. It is shown in Ref. ${ }^{1}$ that the resolvent of $K_{\mathrm{s}}$ may be calculated by Born approximation if no narrow single-particle resonance is present. In the opposite case, one can separate $K_{s}$ or $V_{s}$ into two parts

$$
K_{s}=K_{s}^{\text {sep }}+K_{s}^{\text {rest }}=\frac{1}{E^{+}-H_{0}}\left(V_{s}^{\text {sep }}+V_{s}^{\text {rest }}\right),
$$

where $K_{s}^{\text {sep }}$ is chosen to be operator of finite rank and to contain the most of the effect of the single-particle resonance. The scheme proposed by Weinberg ${ }^{6}$ and applied by the Heidelberg group ${ }^{7}$ consists in taking the following form for $V_{s}^{\text {sep }}$

$$
V_{s}^{\text {sep }}=\frac{V_{s}|W(E)\rangle\langle\tilde{W}(E)| V_{s}}{\left\langle\tilde{W}(E)\left|V_{s}\right| W(E)\right\rangle}
$$

The functions $W(E)$ and $W(E)$ are defined by:

$$
\begin{aligned}
& W(E)=\mathscr{A}\left\{\theta_{l j}\left(r_{A}, k_{c}\right) \varphi_{c}\right\}, \\
& \tilde{W}(E)=\mathscr{A}\left\{\theta_{l j}^{*}\left(r_{A}, k_{c}\right) \varphi_{c}\right\} .
\end{aligned}
$$

The function $\varphi_{c}$ is the surface wave function in channel $c$. The operator $\mathscr{A}$ is the antisymmetrizing operator. The function $\theta_{l j}$ must be such that

$$
\theta_{l j}\left(r, k_{0}^{c}\right)=w_{l j}\left(r, k_{0}^{c}\right) \text {. }
$$

6 Weinberg, S.: Phys. Rev. 130, 776 (1963); 131, 440 (1964).

7 Ebenhöh, W., Glöckle, W., Hüfner, J., Weidenmüller, H. A.: Z. Physik 202, 302 (1967). 
The quantity $k_{0}^{c}$ is the complex wave number corresponding to the resonance, and the function $w_{l j}$ is the Gamow function. Then the Born serie for $K_{s}^{\text {rest }}$ will converge rapidly. If the resonance is broad, it is still expected that the Born serie for $K_{s}^{\text {rest }}$ will converge more rapidly than the one for $K_{s}$.

These considerations will help us to construct GOMP(c). For simplicity, we suppose that we have a single-particle resonance in only the channel $c$. We do not make any assumption in the width of the resonance. With the definitions:

$$
\begin{gathered}
\left\langle\tilde{W}(E)\left|V_{s}\right| \chi_{E^{\prime}}^{c^{\prime}}\right\rangle=V_{M+1}^{c^{\prime}}\left(E^{\prime}\right), \\
\sum_{c^{\prime \prime} \varepsilon} \int_{c^{\prime \prime}}^{\infty} d E^{\prime \prime} \frac{\left\langle\chi_{E^{\prime \prime}}^{c^{\prime \prime}}\left|V_{s}\right| W(E)\right\rangle}{\left\langle\tilde{W}(E)\left|V_{s}\right| W(E)\right\rangle} a_{E}^{c}\left(E^{\prime \prime}, c^{\prime \prime}\right)=b_{E}^{c}(M+1),
\end{gathered}
$$

the Eqs. (2.6) and (2.23) become

$$
\begin{gathered}
\sum_{j=1}^{M+1} B_{i j} b_{E}^{c}(j)=\sum_{c^{\prime} \varepsilon_{c^{\prime}}}^{\infty} d E^{\prime} V_{i}^{c^{\prime}}\left(E^{\prime}\right) a_{E}^{c}\left(E^{\prime}, c^{\prime}\right), \\
\left(E-E^{\prime}\right) a_{E}^{c}\left(E^{\prime}, c^{\prime}\right)-\sum_{m=1}^{M+1} V_{m}^{c^{\prime}}\left(E^{\prime}\right) b_{E}^{c}(m) \\
-\sum_{c^{\prime \prime}} \int_{\varepsilon_{c}^{\prime \prime}}^{\infty} d E^{\prime \prime} V_{s}^{\mathrm{rest}}\left(E^{\prime \prime}, E^{\prime}, c^{\prime \prime}, c^{\prime}\right) a_{E}^{c}\left(E^{\prime \prime}, c^{\prime \prime}\right)=0,
\end{gathered}
$$

if

$$
B_{i j}=\left(\begin{array}{cc}
\left(E-E_{i}\right) \delta_{i j}-V_{i j} & 0 \\
0 & \left\langle\tilde{W}(E)\left|V_{s}\right| W(E)\right.
\end{array}\right)
$$

For $c^{\prime} \neq c$, Eq. (2.25) gives

$$
\begin{aligned}
a_{E}^{c}\left(E^{\prime}, c^{\prime}\right)= & \frac{1}{E^{+}-E^{\prime}}\left\{\sum_{j=1}^{\mathrm{M}+1} V_{j}^{c^{\prime}}\left(E^{\prime}\right) b_{E}^{c}(j)\right. \\
& \left.+\int_{\varepsilon_{c}}^{\infty} d E^{\prime \prime} a_{E}^{c}\left(E^{\prime \prime}, c\right) V_{s}^{\mathrm{rest}}\left(E^{\prime \prime}, E^{\prime}, c, c^{\prime}\right)\right\}+\frac{1}{E^{+}-E^{\prime}} \\
& \cdot\left\{\sum_{c^{\prime \prime} \neq c} \int_{\varepsilon_{c^{\prime \prime}}}^{\infty} d E^{\prime \prime} a_{E}^{c}\left(E^{\prime \prime}, c^{\prime \prime}\right) V_{s}^{\mathrm{rest}}\left(E^{\prime \prime}, E^{\prime}, c^{\prime \prime}, c^{\prime}\right)\right\} .
\end{aligned}
$$

Using the first order Born approximation and eliminating the coefficients $a_{E}^{c}\left(E^{\prime}, c^{\prime}\right)$ for $c^{\prime} \neq c$ and $b_{E}^{c}(j)$ in Eq. (2.24a) and (2.26) we obtain 
Eq. (2.10) with now

$$
\begin{aligned}
\mathscr{V}_{E}^{c}\left(E^{\prime}, E^{\prime \prime}\right)= & \mathscr{V}_{E}^{c(\mathrm{dir})}\left(E^{\prime}, E^{\prime \prime}\right) \\
& +\sum_{j, m=1}^{M+1} W_{E, j}^{c}\left(E^{\prime}\right)\left[{ }_{s}^{c} D^{-1}(E)\right]_{j m} W_{E, m}^{c}\left(E^{\prime \prime}\right), \\
\mathscr{V}_{E}^{c(\mathrm{dir})}\left(E^{\prime}, E^{\prime \prime}\right)= & \left\langle\chi_{E^{\prime}}^{c}\right| V_{s}^{\mathrm{rest}}+V_{s}^{\mathrm{rest}} \sum_{c^{\prime} \neq c} G_{0}^{c^{\prime}}(E) V_{s}^{\mathrm{rest}} \\
& +V_{s}^{\mathrm{rest}} \sum_{c^{\prime} \neq c} G_{0}^{c^{\prime}}(E) V_{s}^{\mathrm{rest}} \sum_{c^{\prime \prime} \neq c} G_{0}^{c^{\prime \prime}}(E) V_{s}^{\text {rest }}\left|\chi_{E^{\prime \prime}}^{c}\right\rangle, \\
W_{E, j}^{c}\left(E^{\prime}\right)= & \left\langle\Phi_{j}\right| V+V \sum_{c^{\prime} \neq c} G_{0}^{c^{\prime}}(E) V_{s}^{\text {rest }} \\
& +V \sum_{c^{\prime} \neq c} G_{0}^{c^{\prime}}(E) V_{s}^{\text {rest }} \sum_{c^{\prime \prime} \neq c} G_{0}^{c^{\prime \prime}}(E) V_{s}^{\text {rest }}\left|\chi_{E^{\prime}}^{c}\right\rangle, \\
{\left[{ }_{s}^{c} D(E)\right]_{j m}=} & B_{j m}-\left\langle\Phi_{j}\right| V \sum_{c^{\prime} \neq c} G_{0}^{c^{\prime}}(E) V \\
& +V \sum_{c^{\prime} \neq c} G_{0}^{c^{\prime}}(E) V \sum_{c^{\prime \prime} \neq c} G_{0}^{c^{\prime \prime}}(E) V\left|\Phi_{m}\right\rangle .
\end{aligned}
$$

If the resonance is present in a channel $c_{0} \neq c$, the formula derived above remains valid, provided one uses the following definition for $W(E)$ :

$$
W(E)=\mathscr{A}\left\{\theta_{l j}\left(r_{A}, k_{c_{0}}\right) \varphi_{c_{0}}\right\} .
$$

\section{The OMP(c)}

By definition, the $\operatorname{OMP}(c)$ is a one-body potential the scattering function of which is equal to the average diagonal element of the collision matrix in channel $c$. If we denote by $\tilde{\rho}_{E}^{c}(r)$ an eigenfunction of $\operatorname{OMP}(c)$, we have in analogy with Eq. (2.14)

$$
\tilde{\rho}_{E}^{c}(r)=\int_{\varepsilon_{c}}^{\infty} d E^{\prime} \tilde{a}_{E}^{c}\left(E^{\prime}, c\right) u_{l j}\left(r, k_{c^{\prime}}\right) .
$$

We have thus to find a quantity $\tilde{\mathscr{V}}_{E}^{c}\left(E^{\prime}, E^{\prime \prime}\right)$ satisfying

$$
\left(E-E^{\prime}\right) \tilde{a}_{E}^{c}\left(E^{\prime}, c\right)-\int_{\varepsilon_{c}}^{\infty} \tilde{\mathscr{V}}_{E}^{c}\left(E^{\prime}, E^{\prime \prime}\right) \tilde{a}_{E}^{c}\left(E^{\prime}, c\right) d E^{\prime \prime}=0 .
$$

This quantity is not unique. If no narrow single-particle resonance is present in channel $c$, i.e. if $\delta_{c}(E+i I) \simeq \delta_{c}(E)$ and $V_{i}^{c}(E+i I) \simeq V_{i}^{c}(E)$, the construction of a possible value of $\tilde{\mathscr{V}}_{E}^{c}$ is simple ${ }^{1}$. One can verify that, for $V_{s}=0$, the quantity defined by

$$
\tilde{\mathscr{V}}_{E}^{c}\left(E^{\prime}, E^{\prime \prime}\right)=\sum_{j, m=1}^{M} V_{j}^{c}\left(E^{\prime}\right)\left[{ }^{c} D^{-1}(E+i I)\right]_{j m} V_{m}^{c}\left(E^{\prime \prime}\right)
$$


satisfies the Eq. (3.2). For $V_{s} \neq 0$, if the single-particle resonance isolated by the method of Section 2 is broad, or more precisely, if its width is larger than the interval $I$, one has

$$
\begin{aligned}
\tilde{\mathscr{V}}_{E}^{c}\left(E^{\prime}, E^{\prime \prime}\right)= & \mathscr{r}_{E}^{c(\mathrm{dir})}\left(E^{\prime}, E^{\prime \prime}\right) \\
& +\sum_{j, m=1}^{M+1} W_{E+i I, j}^{c}\left(E^{\prime}\right)\left[{ }_{s}^{c} D^{-1}(E+i I)\right]_{j m} W_{E+i I, m}^{c}\left(E^{\prime \prime}\right) .
\end{aligned}
$$

In $r$-representation, the Eq. (3.2) reads

$$
\left(E-D_{l j}\right) \tilde{\rho}_{E}^{c}(r)-\int_{0}^{\infty} d r^{\prime} \tilde{\mathscr{r}}_{E}^{c}\left(r, r^{\prime}\right) \tilde{\rho}_{E}^{c}\left(r^{\prime}\right)=0 .
$$

Because of the very definition of the $\operatorname{OMP}(c)$, the latter is given by

$$
\mathscr{\mathscr { V }}_{E}^{\mathrm{opt}(c)}\left(r, r^{\prime}\right)=v_{0}(r)+\tilde{\mathscr{V}}_{E}^{c}\left(r, r^{\prime}\right),
$$

where the potential $v_{0}(r)$ is the shell-model potential contained in $D_{l j}$. In Ref. ${ }^{1}$, it is shown that

$$
\tilde{\mathscr{V}}_{E}^{c}\left(r, r^{\prime}\right)=\int_{\varepsilon_{c}}^{\infty} d E^{\prime} \int_{E_{c}}^{\infty} d E^{\prime \prime} u_{l j}\left(r, k_{c}^{\prime}\right) \tilde{\mathscr{V}}_{E}^{c}\left(E^{\prime}, E^{\prime \prime}\right) u_{i j}\left(r, k_{c}^{\prime \prime}\right) .
$$

\section{Energy Dependence of $\mathrm{OMP}(c)$ in the Presence of a Broad Single-Particle Resonance}

As we mentioned in the introduction, we only study the dynamic energy dependence of $\operatorname{OMP}(c)$, i.e. the one obtained by keeping the "natural" non-locality introduced by the shell-model calculation of the optical-model potential. We show that $\mathrm{OMP}(c)$ is nearly energy independent in the vicinity of a broad single-particle resonance, even when direct processes are taken into account. The consequence of those processes is to add real and imaginary terms in $\mathrm{OMP}(c)$ which are slowly varying with energy. However, the optical-model phase shift exhibits a resonance. We explain this apparent contradiction by means of a simple relation between $\operatorname{OMP}(c)$ and the optical model phase shift, that we derive in the beginning of this section. This relation emphasizes the fact that both real and imaginary parts of OMP $(c)$ contribute to the imaginary part of the optical-model phase shift.

We start with the calculation of $\operatorname{OMP}(c)$ in a very simple case: no single-particle resonance, $V_{s}=0$. Let us remind the form of the matrix ${ }^{c} D(E+i I)$ :

$$
\begin{aligned}
& {\left[{ }^{c} D(E+i I)\right]_{j m}} \\
& \quad=\left(E+i I-E_{j}\right) \delta_{j m}-V_{j m}-\sum_{c^{\prime} \neq c \varepsilon_{\varepsilon^{\prime}}} \int^{\infty} d E^{\prime} \frac{V_{j}^{c^{\prime}}\left(E^{\prime}\right) V_{m}^{c^{\prime}}\left(E^{\prime}\right)}{E+i I-E^{\prime}} .
\end{aligned}
$$


If we assume that the quantities $V_{j m}$ and $\sum_{c^{\prime} \neq c} \int_{\varepsilon_{c^{\prime}}}^{\infty} d E^{\prime} \frac{V_{j}^{c^{\prime}}\left(E^{\prime}\right) V_{m}^{c^{\prime}}\left(E^{\prime}\right)}{E+i I-E^{\prime}}$ have random signs and that their contributions vanish, we can neglect the off-diagonal terms in the matrix ${ }^{c} D(E+i I)$.

The optical-model potential is then given by:

$$
\mathscr{V}_{E}^{\mathrm{opt}(c)}\left(r, r^{\prime}\right)=v_{0}(r)+\sum_{j=1}^{M} \frac{V_{j}^{c}(r) V_{j}^{c}\left(r^{\prime}\right)}{E+i I-E_{j}}
$$

Since the dispersion of the quantities $V_{j}^{c}(r) V_{j}^{c}\left(r^{\prime}\right)$ and $E_{j+1}-E_{j}$ is not large, the second term in the r.h.s. of Eq. (4.2) is approximately equal to $-i \mathscr{S}_{c}\left(r, r^{\prime}\right)$, with

$$
\begin{aligned}
\mathscr{S}_{c}\left(r, r^{\prime}\right) & =\frac{\pi}{d}{\overline{V_{j}^{c}(r) V_{j}^{c}\left(r^{\prime}\right)^{j}}}^{j}, \\
d & ={\overline{E_{j+1}-E_{j}}}^{j} .
\end{aligned}
$$

The bar means an average over the indice $j$. Thus we have

$$
\mathscr{V}_{E}^{\text {opt }(c)}\left(r, r^{\prime}\right)=v_{0}(r)-i \pi \mathscr{S}_{c}\left(r, r^{\prime}\right) \text {. }
$$

In this case, there exists a simple relation between characteristic quantities of the optical model. Let us call $\delta_{c}^{\text {opt }}$ the phase shift due to $\mathscr{V}_{E}^{\text {opt }(c)}\left(r, r^{\prime}\right)$. We have in first order in $\mathscr{S}_{c}\left(r, r^{\prime}\right)^{8}$ :

or

$$
\delta_{c}^{\mathrm{opt}} \simeq \delta_{c}-\pi \int_{0}^{\infty} d r \int_{0}^{\infty} d r^{\prime} u_{c}(r, k)\left[-i \mathscr{S}_{c}\left(r, r^{\prime}\right)\right] u_{c}\left(r^{\prime}, k\right)
$$

$$
\delta_{c}^{\mathrm{opt}} \simeq \delta_{c}+i \pi S_{c}(E)
$$

The function $S_{c}(E)$ is called the "strength function in channel $c$ " and is defined by:

$$
S_{c}(E)=\frac{\overline{\pi\left[V_{j}^{c}(E)\right]^{2} j}}{d}
$$

The quantity $\delta_{c}^{\text {opt }}$ is the optical model phase shift and can be obtained from the average total cross section in channel $c$. Since the $\operatorname{OMP}(c)$ leads to the average diagonal element $S_{c c}$ of the collision matrix in channel $c$, we must have

$$
\exp \left(i 2 \delta_{c}^{\mathrm{opt}}\right)=\left\langle S_{c c}(E)\right\rangle
$$

8 Messiah, A.: Quantum mechanics, p. 363. Dunod 1964. 
Let us show it explicitly. From Eq. (2.7), we get

$$
\begin{aligned}
\left\langle S_{c c}(E)\right\rangle= & \exp \left(i 2 \delta_{c}\right) \\
& \cdot\left\{1-i 2 \pi \sum_{j, m} V_{j}^{c}(E)\left[D^{-1}(E+i I)\right]_{j m} V_{m}^{c}(E)\right\} .
\end{aligned}
$$

By the same method as above, we have:

$$
\left\langle S_{c c}(E)\right\rangle=\exp \left(2 i \delta_{c}\right)\left\{1-2 \pi \frac{\overline{\pi\left[V_{j}^{c}(E)\right]^{2}}}{d}\right\}
$$

or, in the first order in the strength function, with the help of Eq. (4.6b):

$$
\left\langle S_{c c}(E)\right\rangle \simeq \exp \left\{2 i\left[\delta_{c}+i \pi S_{c}(E)\right]\right\}_{\text {; }}
$$

There exists also a simple relation between the "strength function" and the transmission coefficient $T_{c}$ defined by:

$$
T_{c}=1-\left|\left\langle S_{c c}(E)\right\rangle\right|^{2} .
$$

Here, one gets because of Eq. ( $4.9 \mathrm{~b})$

$$
T_{c} \simeq 1-\exp \left(-4 \pi S_{c}(E)\right)
$$

or, because of Eq. (4.9a)

$$
T_{c}=1-4 \pi S_{c}(E)+4 \pi^{2}\left[S_{c}(E)\right]^{2} .
$$

The Eqs. (4.9b) and (4.11 a) agree with the results of Moldauer ${ }^{9}$ provided the strength function in channel $c$ is small. Then, the formulae (4.9b) and $\left(4.11\right.$ a) are a good approximation. When the assumption $S_{c} \ll 1$ is removed, the Eqs. (4.9a) and (4.11b) remain valid. This is not the case for Eqs. (4.9b) and (4.11a). The calculations of Moldauer show that they are still valid if one takes the following definition of $S_{c}$ :

$$
S_{c}(E)=\frac{\overline{\Gamma_{j}^{c}} j}{d} .
$$

Let us recall those formulae have been derived in the absence of direct processes and potential resonances. After this simple case, we turn to more and more complicated ones.

\section{$V_{s}=0$. Presence of a Broad Single-Particle Resonance}

If the single-particle resonance is present in channel $c$, the matrix ${ }^{c} D$ remains the same and the optical-model potential is still given by Eq. (4.4). However, the optical phase shift may be different because of the presence

9 Moldauer, P. A.: Phys. Rev. 157, 907 (1967); 171, 1164 (1968). 
of $G_{0}^{c}$ in $D$. One has:

$$
[D(E+i I)]_{j m} \simeq\left(E+i I-E_{j}\right) \delta_{j m}-\left\langle\Phi_{j}\left|V G_{0}^{c}(E+i I) V\right| \Phi_{m}\right\rangle .
$$

The Green function $G_{0}^{c}(E)$ may be approximated by $\frac{\left.W_{0}^{c}\right\rangle\left\langle W_{0}^{c}\right.}{E-\mathscr{E}_{0}^{c}}$. The function $W_{0}^{c}$ is the many-body Gamow function, and the quantity $\mathscr{E}_{0}^{c}$ is the complex energy of the resonance:

Hence, one has

$$
\mathscr{E}_{0}^{c}=E_{0}-i \Gamma_{0} / 2=\varepsilon_{c}+\frac{\hbar^{2}\left(k_{0}^{c}\right)^{2}}{2 M_{c}}
$$

$$
\left\langle\Phi_{j}\left|V G_{0}^{c}(E+i I) V\right| \Phi_{m}\right\rangle \simeq \frac{\left\langle\Phi_{j}|V| W_{0}^{c}\right\rangle\left\langle W_{0}^{c}|V| \Phi_{m}\right\rangle}{E-\mathscr{E}_{0}^{c}} .
$$

If one assumes that, for all $j$ and $m$,

$$
\left\langle\Phi_{j}|V| W_{0}^{c}\right\rangle^{2}=\left\langle W_{0}^{c}|V| \Phi_{m}\right\rangle^{2}=W^{2 \star},
$$

the matrix $D(E+i I)$ becomes:

$$
[D(E+i I)]_{j m}=\left(E+i I-E_{j}\right) \delta_{j m}-\frac{W^{2}}{E-\mathscr{E}_{0}^{c}} \mathscr{I} .
$$

The matrix $\mathscr{I}$ is symmetric. Its diagonal elements are +1 and its non diagonal ones are statistically +1 or -1 . Because of the randomness in the signs of the off-diagonal matrix elements, we may assume that they have mutually cancellating effects. So, the optical phase shift keeps the same form (4.6a) in spite of the presence of the single-particle resonance. But the values of the strength function is peaked at $E_{0}$ because the value of $\left(V_{j}^{c}\right)^{2}$ is also increasing in the vicinity of the s.-p. resonance.

If the resonance is present in a channel $c_{0} \neq c$, we have to keep the terms $\left\langle\Phi_{j}\left|V G_{0}^{c_{0}}(E+i I) V\right| \Phi_{m}\right\rangle$ in ${ }^{c} D(E+i I)$ for the calculation of the optical-model potential. With the help of arguments similar to those given above, one obtains again the formula (4.4) for the OMP(c) and (4.6a) for the optical model phase shift. But, now $\operatorname{Im} \delta_{\text {opt }}^{(c)}$ does not display any resonance.

\section{One Broad Single-Particle Resonance. $V_{s} \neq 0$}

When a broad single-particle resonance exists in channel $c$, the matrix ${ }_{s}^{c} D(E+i I)$ is given by:

$$
{ }_{s}^{c} D(E+i I) \simeq\left(\begin{array}{cc}
\left(E+i I-E_{j}\right) \delta_{j m} & 0 \\
0 & \left\langle\tilde{W}(E)\left|V_{s}\right| W(E)\right\rangle
\end{array}\right),
$$

* The matrix elements $\left\langle\Phi_{j}|V| W_{0}^{c}\right\rangle$ are not exactly real. They have an imaginary component, which is small provided one has: $\operatorname{Im} k_{0}^{c} \ll \operatorname{Re} k_{0}^{c}$. 
since all the terms containing the Green functions are negligible for $c^{\prime} \neq c$ and for $\mathscr{E}=E+i I$. Proceeding like in the preceeding cases, one finds:

$$
\begin{aligned}
\mathscr{V}_{E}^{\mathrm{opt}(c)}\left(r, r^{\prime}\right) & =v_{0}(r)+\mathscr{V}_{E}^{c(\mathrm{dir})}\left(r, r^{\prime}\right)+\sum_{j=1}^{M} \frac{W_{j}^{c}(r) W_{j}^{c}\left(r^{\prime}\right)}{E+i I-E_{j}} \\
& +\frac{W_{M+1}^{c}(r) W_{M+1}^{c}\left(r^{\prime}\right)}{\left\langle\tilde{W}(E)\left|V_{s}\right| W(E)\right\rangle} .
\end{aligned}
$$

The quantities $\mathscr{V}_{E}^{c \text { (dir) }}\left(r, r^{\prime}\right)$ and $W_{j}^{c}(r)$ are the transforms by the functions $u_{l j}(r, k)$ of the quantities $\mathscr{V}_{E}^{c}{ }^{\text {(dir) }}\left(E^{\prime}, E^{\prime \prime}\right)$ and $W_{E j}^{c}\left(E^{\prime}\right)$ defined in Section 2. The quantities $W_{j}^{c}(r)$ depend on $E$, because of the energydependence of the functions $W_{E j}^{c}\left(E^{\prime}\right)$. Since they are slowly varying, we neglect here this dependence. Using a picket fence model for thecompound nucleus states and taking account of Eq. (2.22), we get:

$$
\begin{aligned}
\mathscr{V}_{E}^{\mathrm{opt}(c)}\left(r, r^{\prime}\right)= & v_{0}(r)+\mathscr{V}_{E}^{c(\mathrm{dir})}\left(r, r^{\prime}\right)-i \pi \frac{\overline{W_{j}^{c}(r) W_{j}^{c}\left(r^{\prime}\right)^{j}}}{d} \\
& +\frac{W_{M+1}^{c}(r) W_{M+1}^{c}\left(r^{\prime}\right)}{\left\langle\tilde{W}\left|V_{s}\right| W\right\rangle} .
\end{aligned}
$$

The term $v_{0}(r)$ accounts for the potential scattering and particularly for the single-particle resonance. The second term corresponds to direct phenomena governed by the part $V_{s}^{\text {(rest) }}$ of $V_{s}$ which does not contain the single-particle resonance. Since the second order in $V_{s}^{\text {(rest) }}$, the

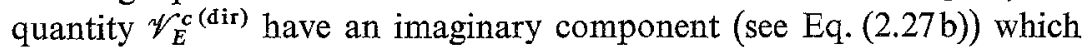
is due to the absorption by the other channels. The absorption by the compound nucleus states is represented by the quantity

$$
\mathscr{S}_{c}\left(r, r^{\prime}\right)=\pi \frac{\overline{W_{j}^{c}(r) W_{j}^{c}\left(r^{\prime}\right)^{j}}}{d} .
$$

Its transform $\mathscr{S}_{c}(E)$ by the functions $u_{c}(r, k)$ is defined by:

$$
\mathscr{S}_{c}(E)=\frac{\pi}{d} \cdot{\overline{\left[W_{j}^{c}(E)\right]^{2}}}^{j}
$$

and is a generalization of the strength function. The quantities $W_{j}^{c}(E)$ are substituted to the $V_{j}^{c}$ 's because processes like

$$
\begin{array}{ll}
\chi_{E}^{c} \rightarrow \Phi_{j} \rightarrow \chi_{E}^{c^{\prime}} \rightarrow \chi_{E}^{c} & \text { (1st order) } \\
\chi_{E}^{c} \rightarrow \Phi_{j} \rightarrow \chi_{E}^{c^{c^{\prime}}} \rightarrow \chi_{E}^{c^{\prime \prime}} \rightarrow \chi_{E}^{c} & \text { (2nd order) }
\end{array}
$$

are now present. The fourth term of Eq. (4.20) represents the part due to the interference of the single-particle resonance with the direct processes. This is a slowly varying function of the energy. 
If the single-particle resonance takes place in channel $c_{0} \neq c$, the matrix ${ }_{s}^{c} D(E+i I)$ can be put in the form

$$
{ }_{s}^{c} D(E+i I) \simeq\left(\begin{array}{cc}
\left(E+i I-E_{j}\right) \delta_{j m} & 0 \\
0 & \left\langle\tilde{W}(E)\left|V_{s}\right| W(E)\right\rangle
\end{array}\right)
$$

with the help of the same arguments than those described above, provided the matrix elements involving $W(E)$ are of the same order of magnitude than the same matrix elements with the functions $\Phi_{j}$. Again, one gets Eq. (4.20) for the optical-model potential.

An alternative way of deriving the form of the optical-model potential consists in treating $V_{s}$ as a whole without extracting the part responsible of the effect of resonance. If the latter is broad enough, $V_{s}$ can be treated by the Born serie. In first order in $V_{s}$, one gets:

$$
\hat{\mathscr{V}}_{E}^{\text {opt(c) }}\left(r, r^{\prime}\right)=v_{0}(r)-i \hat{\mathscr{S}}_{c}\left(r, r^{\prime}\right)+\hat{\mathscr{V}}_{E}^{c(\mathrm{dir})}\left(r, r^{\prime}\right) .
$$

The quantities $\hat{\mathscr{S}}_{c}$ and $\mathscr{\mathscr { V }}_{E}^{c \text { (dir) }}$ are defined like the similar quantities without hat provided $V_{s}$ is substituted to $V_{s}^{\text {rest }}$. The two expressions (4.20) and (4.25) for the $\operatorname{OMP}(c)$ are equivalent, when calculated exactly, but differ at each order of the Born serie. It is expected that the serie converges more rapidly in the first case than in the second one, at least in the vicinity of the resonance.

\section{Conclusions}

We derived the formula for GOMP(c) obtained in the frame of the shell-model reaction theory, when the channel-channel coupling $V_{s}$ is zero. The formula remains valid in the presence of a single-particle resonance. We derived a formula for GOMP $(c)$ when $V_{s} \neq 0$. The effect of a single-particle resonance in $V_{s}$ is extracted by a method suggested by Weinberg. The remaining part of $V_{s}$, i.e. $V_{s}^{\text {rest }}$ is treated in first order Born approximation. In Section 3, we derived the formulae for OMP(c) in both cases $V_{s}=0$ and $V_{s} \neq 0$ in the presence of a broad single-particle resonance whose width is larger than the average interval $I$. In this case, $\operatorname{OMP}(c)$ is GOMP $(c)$ evaluated at the complex energy $E+i I$. In Section4, we studied the energy behaviour of $\operatorname{OMP}(c)$ through a broad singleparticle resonance. We started with the simplest case: $V_{s}=0$, no singleparticle resonance. No energy dependence is found in the optical-model potential. We also derived a relation between the imaginary part of OMP $(c)$, the "strength function" $S_{c}$ in channel $c$ and the optical model phase shift $\delta_{c}^{\text {(opt) }}$. When a broad single-particle resonance is present, the optical-model potential is still energy-independent, while the imaginary part of the optical model phase shift (and also $S_{c}$ ) displays a resonance. This situation is due to the fact that the imaginary part of the optical 
phase shift depends upon both the real and the imaginary parts of $\operatorname{OMP}(c)$ as exhibited by Eq. (4.6). Hence, the absorption displays a resonance while the $\mathrm{OMP}(c)$ is constant as far as the dynamic energy dependence is concerned. If the channel-channel coupling is introduced, two modifications arise: i) the "strength function" must be defined somewhat differently. ii) Two new terms appear in $\operatorname{OMP}(c)$. The first one describes the effect of the single-particle resonance in the direct processes. The second one corresponds to direct phenomena due to $V_{s}^{\text {rest }}$. It has an imaginary component due to the fact that absorption by other channels is allowed. Both these terms are slowly energy dependent.

We want to thank Prof. C. Mahaux for helpful discussions and a careful reading of the manuscript.

Dr. J. Cugnon

Université de Liège

Physique Nucléaire Théorique

Institut de Physique

Sart Tilman

Liège 1, Belgique 